\title{
Infantile Convulsions and Paroxysmal Choreoathetosis, Familial
}

National Cancer Institute

\section{Source}

National Cancer Institute. Infantile Convulsions and Paroxysmal Choreoathetosis,

Familial. NCI Thesaurus. Code C126650.

An autosomal dominant inherited disorder characterized by epileptic seizures and paroxysmal kinesigenic choreoathetosis. 